\title{
Etude de systèmes de radiocommunications
}

\author{
Plateforme de tests et de validation des modèles de simulation
}

\author{
Jacques VERDIER, Guillaume VILLEMAUD, Jean-Marie GORCE \\ Départements Télécommunications et Génie Electrique de l'INSA de LYON \\ 20, avenue Albert Einstein 69621 Villeurbanne cedex - France \\ email : jacques.verdier@insa-lyon.fr
}

\section{Résumé}

La complexité des systèmes radio actuels comme futurs, couplée à la nécessité pour un étudiant ou un élève ingénieur, issu du domaine de l'electrical engineering et/ou des télécommunications d'accéder à une formation adaptée à son futur métier, impose aux établissements de se doter de moyens matériels et logiciels ambitieux, à la pointe des nouvelles technologies. Les élèvesingénieurs des départements Génie Electrique et Télécommunications de l'INSA de Lyon disposent maintenant des techniques les plus avancées en conception, modélisation et test de systèmes de radiocommunications. Nous présentons dans cet article une plate-forme transversale dédiée à l'étude de ces systèmes qui permet aux élèves d'appréhender les défis majeurs liés aux nouvelles technologies existantes ou futures qui sont axés principalement sur les performances de l'interface radio (bruit, sensibilité, stabilité, cadence d'échantillonnage) et sur les processus algorithmiques (traitement et analyse du signal à une cadence élevée). Deux sujets pratiques destinés aux étudiants de $5^{\text {ème }}$ année (étudiants INSA) et de $2^{\text {nd }}$ année de MASTER (étudiants hors INSA) sont finalement présentés. Ils traitent pour le premier des algorithmes SIMO appliqués aux récepteurs multistandards et pour le second sujet de l'influence des imperfections des différents blocs radiofréquences d'une liaison radio WLAN (Wireless Local Area Network) 802.11g sur les performances globales.

\section{Introduction}

Au cours des 10 dernières années de nombreuses technologies de transmission sans fil ont vu le jour. Ces technologies [1][2] ont émergé à la fois dans le domaine de la téléphonie mobile (GSM Global System for Mobile Communication, UMTS Universal Mobile Telecom System) et dans le domaine des réseaux locaux sans fil (standards IEEE 802.11, Hiperlan, Bluetooth). Ce fort développement des Technologies de l'Information et de la Communication (TIC) a naturellement amené les universités et les écoles d'ingénieurs à faire évoluer leur formation type EEA. C'est particulièrement le cas dans le secteur des communications sans fil et il apparaît aujourd'hui des filières spécifiques aux noms évocateurs tels que Electronique des Communications ou encore Systèmes Intégrés pour les Radiocommunications. En effet, la transmission de données, parole et vidéo trouvant des applications de plus en plus nombreuses et variées il est devenu nécessaire aux futurs diplômés de comprendre et maîtriser les problèmes liés à la mise en place d'une liaison radio en fonction de l'environnement, de la mise en forme et du débit des données source, de la puissance disponible à l'antenne, de la sélectivité et de sensibilité du récepteur ...

L'innovation technologique a un rôle très important dans le domaine des TIC. Il apparait alors nécessaire aujourd'hui que les formations universitaires ou d'ingénieurs apportent des propositions adaptées et innovantes en matière de pédagogie et d'outils associés tout en maîtrisant au mieux le caractère fondamental des enseignements, seul garant d'une formation solide et pérenne. C'est ainsi, au niveau de l'INSA de Lyon et plus précisément des départements Télécommunications et Génie Electrique que nous avons développé, en collaboration avec la société Agilent Technologies, une plateforme de tests et d'étude des systèmes de radiocommunications et de validation des modèles de simulation. 


\section{Plateforme d'étude des systèmes de radiocommunications}

Le marché grand public des systèmes de communications mobiles connaît un essor considérable depuis près de 15 ans. C'est l'utilisation des technologies radio numériques qui a, par exemple, permis d'améliorer la qualité de la voix et d'augmenter significativement le nombre d'utilisateurs. On peut également souligner que ces progrès technologiques ont favoriser la transmission de données plus complexes et surtout plus volumineuses, telles que les images. A travers la terminologie des «technologies radio » ou "sans fils », on regroupe généralement toutes les caractéristiques de la norme associée au standard étudié. Outre les bandes de fréquence allouées pour les liens montants et descendants ainsi que le nombre de canaux et leur largeur fréquentielle, il s'agit de décrire les mode d'accès (FDM Frequency Division Multiple, OFDM Orthogonal Frequency Division Multiple, TDMA Time Division Multiple Access, FDMA Frequency Division Multiple Access, CDMA Code Division Multiple Access...), d'indiquer le type de multiplexage souvent dénommé duplexage (FDD Frequency Division Duplex, TDD Time Division Duplex), le type de modulation (GMSK Gaussian Minimum Shift Keying, QPSK Quadrature Phase Shift Keying, DQPSK Differential Quadrature Phase Shift Keying, QAM Quadrature Amplitude Modulation...) ou encore le débit binaire brut et le codage de parole. Il est clair aujourd'hui qu'un des défis de ces prochaines années concerne l'interaction entre ces technologies. Les constructeurs travaillent sur des récepteurs multi-normes, par exemple GSM-WiFi, permettant à un seul équipement de se connecter à différents réseaux. Les équipements actuels intègrent pour cela plusieurs interfaces radio. En parallèle, dans ceux qui sont à l'étude, on cherche à développer et à sélectionner les techniques favorisant l'implantation entièrement logicielle d'un émetteur-récepteur radio favorisant ainsi la reconfigurabilité du système. En effet, cette reconfigurabilité est fortement compromise par toutes les parties hardware et notamment les composants et circuits radiofréquences. La complexité de l'étude et de la conception des systèmes de radiocommunications apparaît donc très importante aujourd'hui et nécessite de nouveaux outils logiciels et matériels.

La plateforme radio présentée sur la figure 1 est un outil d'analyse qui permet d'éprouver les techniques les plus avancées en conception, modélisation et test des systèmes de

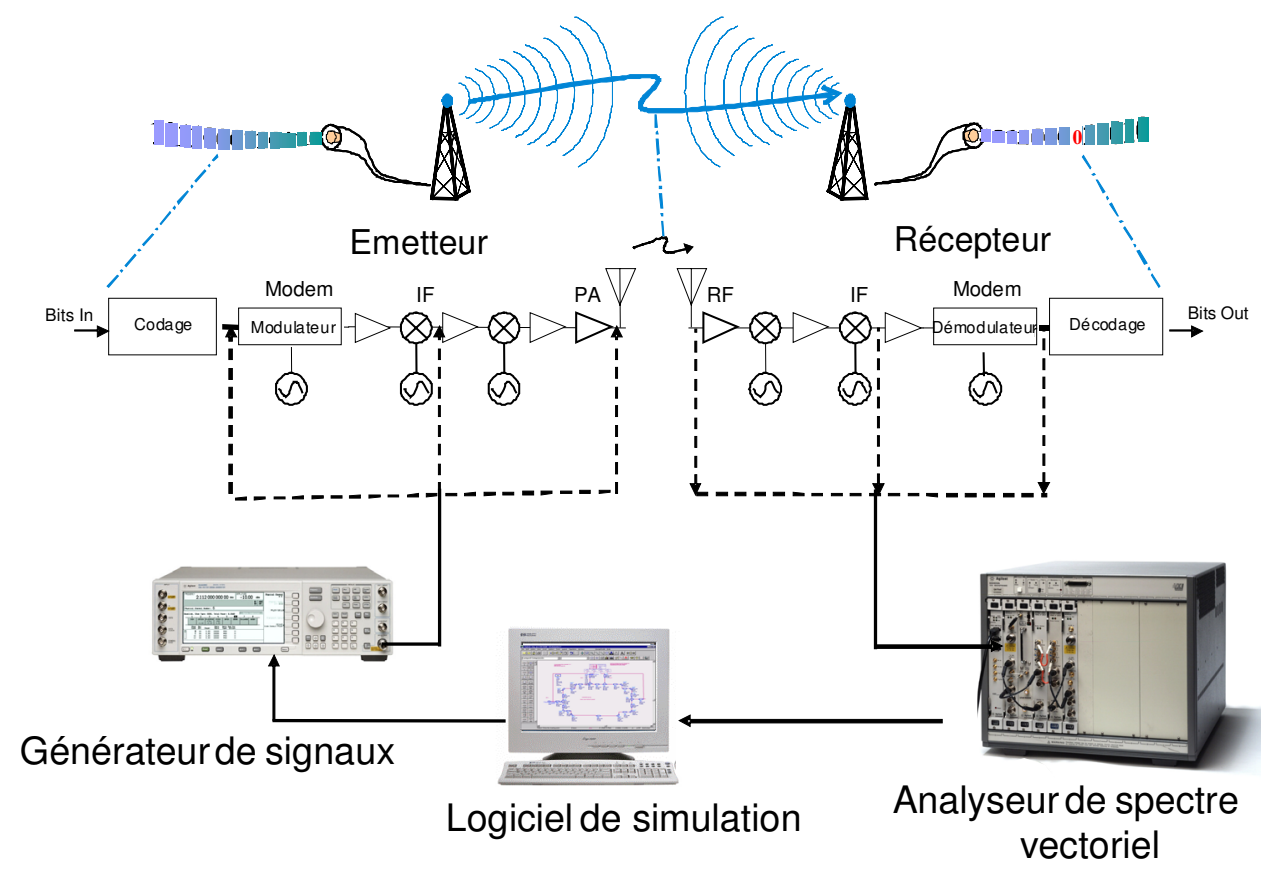

figure 1 : Schéma de la plateforme radio.

radiocommunications. Tous les maillons d'une chaîne d'émission-réception (E/R) peuvent ainsi être étudiés, aussi bien les parties électroniques analogiques, numériques et RF (amplificateurs, 
modulateurs, filtres, antennes, ...) que les aspects et modèles liés à la propagation (canal radio) et bien entendu les techniques de traitement du signal associées.

Cette plateforme est composée d'équipements industriels performants développés par la société Agilent Technologies [3][4] : le logiciel ADS (Advanced Design System) de CAO de circuits RF et microondes, deux générateurs d'onde arbitraire (ESG4438C) et un analyseur de spectre vectoriel (VSA89641) avec 2 entrées RF et des entrées I et Q. La plateforme présente à ce jour une structure $2 \times 2$ voies qui fonctionne jusqu'à $6 \mathrm{GHz}$ et qui possède, au niveau de la réception une bande passante de $36 \mathrm{MHz}$ (70 MHz au niveau du générateur de signaux arbitraires). Ses potentialités résident dans la forte interaction matériel-logiciel qui donne la possibilité d'étudier tout ou partie d'une chaîne E/R c'est à dire les parties RF ou FI (fréquence intermédiaire) ou encore celles en bande de base. En effet, la source d'onde arbitraire est capable de générer n'importe quel signal complexe (signal numérique en bande de base transposé autour de la fréquence RF ou FI jusqu'à $6 \mathrm{GHz}$ ) que l'on peut alors analyser après propagation, dans un environnement réel, avec l'analyseur de spectre vectoriel. Le logiciel d'analyse vectorielle associé peut alors démoduler le signal reçu et offrir différentes options de visualisation comme la densité spectrale de puissance en fonction de la fréquence (le spectre obtenu est alors comparé au spectre d'émission), l'ACPR (Adjacent Channel Power Ratio) qui est définie comme le rapport entre la puissance moyenne dans le canal adjacent en fréquence (gauche ou droit) sur la puissance moyenne dans le canal de transmission, le diagramme de constellation des états, l'EVM (Error Vector Measurement) qui représente les distorsions d'amplitude et de phase sur le diagramme de constellation, le TEB (Taux d'Erreur par Bit)... Généralement, l'optimisation isolée des techniques de transmission (c'est à dire l'étude de certains blocs du système pris séparément du système complet) mène régulièrement à des résultats décevants si la mise en pratique matérielle n'a pas été prise en compte. L'utilisation de cette plateforme est alors un atout véritable puisqu'elle permet une conception globale d'un système à différents niveaux (hardware, traitement du signal) dans un contexte de fonctionnement réaliste. En effet, un signal radio créé sous ADS peut être chargé dans la mémoire interne du générateur ESG puis émis via une antenne. Le signal reçu et mesuré par l'analyseur VSA peut être enregistré et transféré à nouveau sous le logiciel ADS. Cette interaction matériel-logiciel peut se situer en RF, en FI comme au niveau des voies I et $\mathrm{Q}$ ou après le convertisseur Analogique Numérique. Sur la figure 2, nous avons reporté l'exemple où les entrées I et $\mathrm{Q}$ de l'analyseur vectoriel sont utilisées permettant d'étudier la qualité du signal (TEB) entre l'entrée « RF » et la récupération des voies I et $\mathrm{Q}$ du bloc conçu et réalisé.

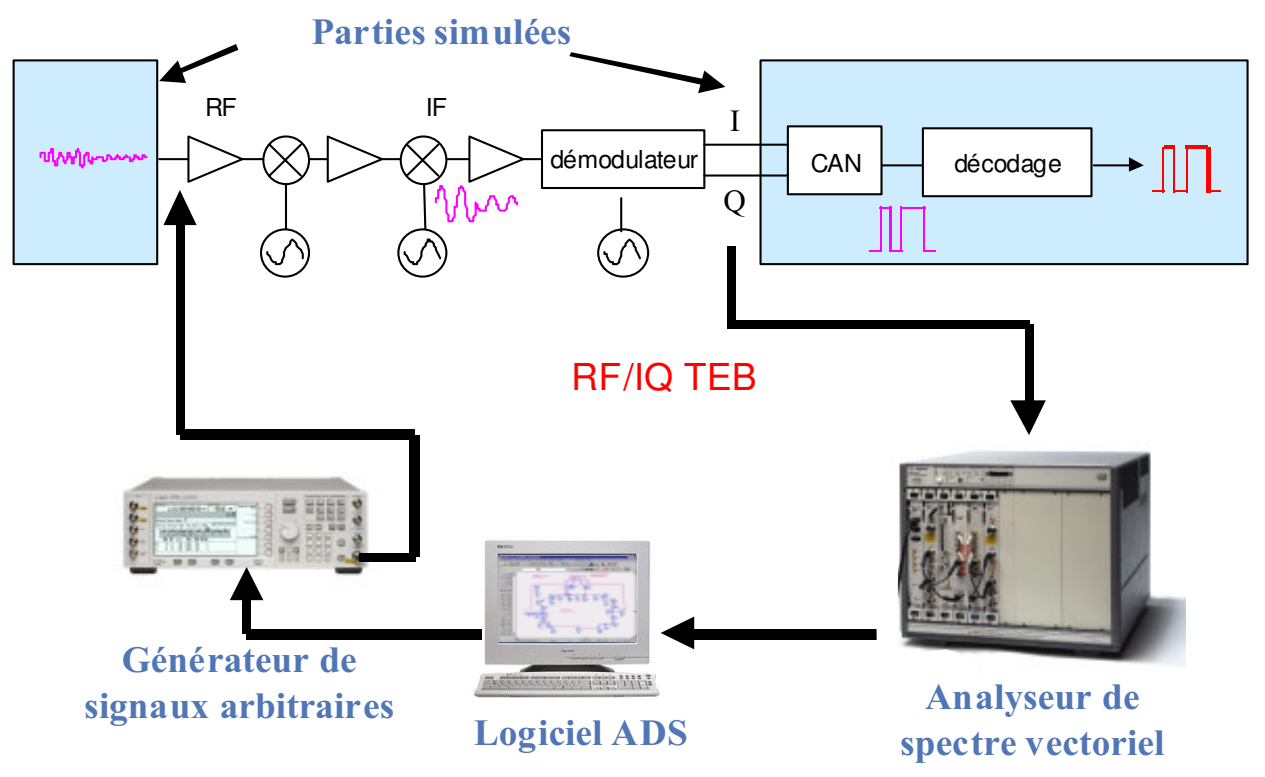

figure 2 : Exemple d'étude « simulations - mesures ». 
Dans le récepteur schématisé, l'émetteur et son signal ont été modélisés sous ADS (simulateur Ptolemy) et émis via l'ESG. Au niveau du récepteur, les parties allant de l'antenne aux signaux I(t) et $\mathrm{Q}(\mathrm{t})$ sont soit des réalisations pour la conception du système ou soit des circuits déjà existants. Le signal de sortie est alors injecté dans le VSA pour être à nouveau traité sous ADS. Sur la figure 3, un second exemple est schématisé où l'on a représenté cette fois-ci la partie émettrice dans laquelle un composant réel de la chaîne est sous test. Via ADS, le signal RF émis par l'ESG comportant les imperfections du modulateur, du mélangeur, du filtre RF... va «attaquer » l'entrée du dispositif " hardware ». Le signal de sortie RF est alors re-injecté dans la chaîne via le VSA. Sur les figures $4 \mathrm{a}$ et $4 \mathrm{~b}$, nous présentons des résultats obtenus dans le cas particulier où le dispositif à tester est un amplificateur de puissance (PA). Nous avons reporté une fenêtre du VSA comportant 4 quadrants, la densité spectrale de puissance (DSP) centrée à $1,95 \mathrm{GHz}$ et pour une bande de fréquence d'analyse (span) de $24 \mathrm{MHz}$, le signal temporel (données numériques), la constellation des états et dans le dernier quadrant quelques caractéristiques relatives au signal, comme l'EVM. Le signal utilisé pour stimuler l'entrée du PA a été créé sous ADS et présente des distorsions en amplitude et un certain niveau d'interférence inter-symboles. Le signal d'entrée présente un EVM de 6,7\%. Le signal en sortie du PA présente un EVM largement augmenté $(8,9 \%)$ ainsi qu'une forte déformation de son spectre fréquentiel. C'est ce signal, tenant compte des imperfections du PA, qui est injecté dans le reste de la chaîne à étudier.

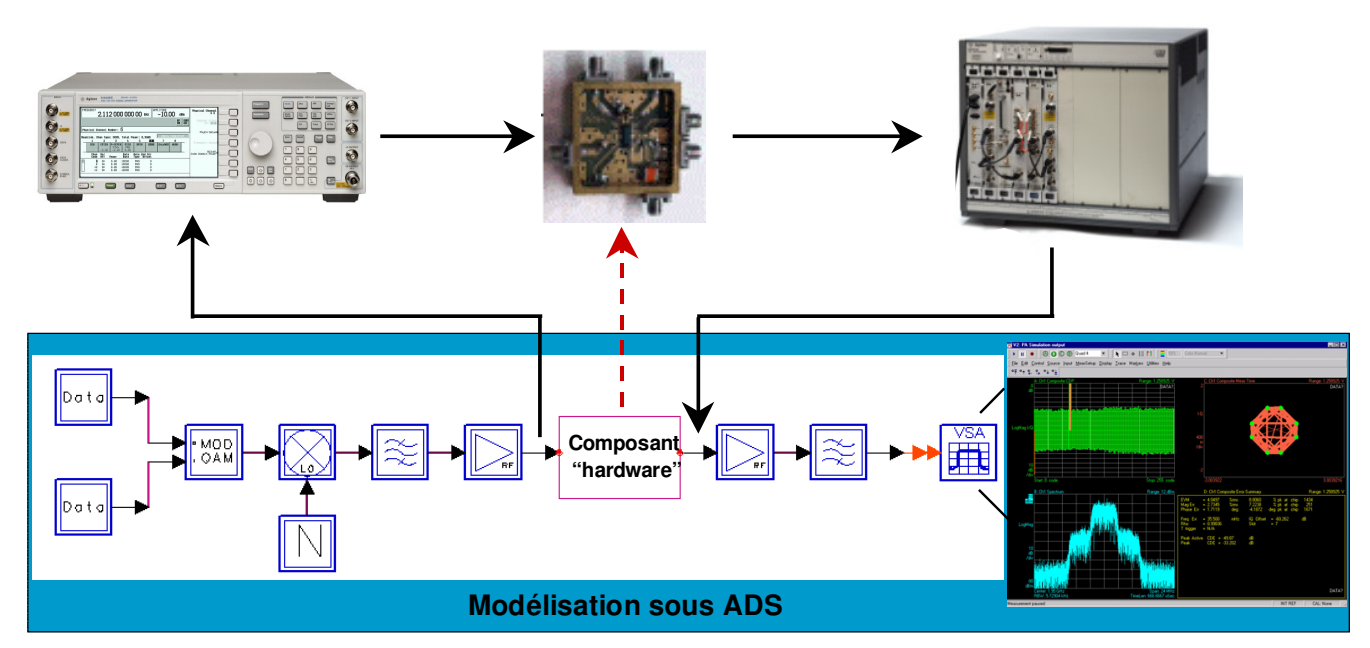

figure 3 : Exemple d'aide à la conception/vérification du système radio.

Enfin, et ce n'est pas le moindre des intérêts de cette plateforme, il est possible, pour accélérer la conception d'un système, de modéliser rapidement sous ADS-Ptolemy via des mesures effectuées sur des analyseurs de réseau ou de spectre, sur des bancs de caractérisation en puissance, des dispositifs tels que des amplificateurs, des filtres, ou encore des oscillateurs qui viennent d'être conçus (mais pas le système dans son ensemble) et de les évaluer dans la chaîne $E / R$. Le canal de propagation également, à partir de tests émission-réception, peut être rapidement modélisé et implanté sous ADS. Sur la figure 5, nous avons reporté une modélisation d'un système WLAN (Wireless Local Area Network) 802.11b dédiée à l'étude des performances en taux d'erreur par bits (TEB) en fonction du rapport signal sur bruit (S/B ou $S N R$ en anglais). La configuration proposée se découpe en trois parties pour permettre la comparaison entre les données mesurées et simulées. En effet, une première partie, où sont repérés la source WLAN, la modélisation du canal et le récepteur donne la possibilité de déterminer le TEB simulé. En dessous, le bloc permettant de charger sur l'ESG le signal à émettre est clairement reporté. Enfin, la troisième partie précise comment le signal reçu et enregistré peut à nouveau être «joué » sous Ptolemy pour extraire cette fois-ci le TEB mesuré. La qualité du modèle de canal utilisé est évidemment gage d'une bonne évaluation des performances en TEB. 


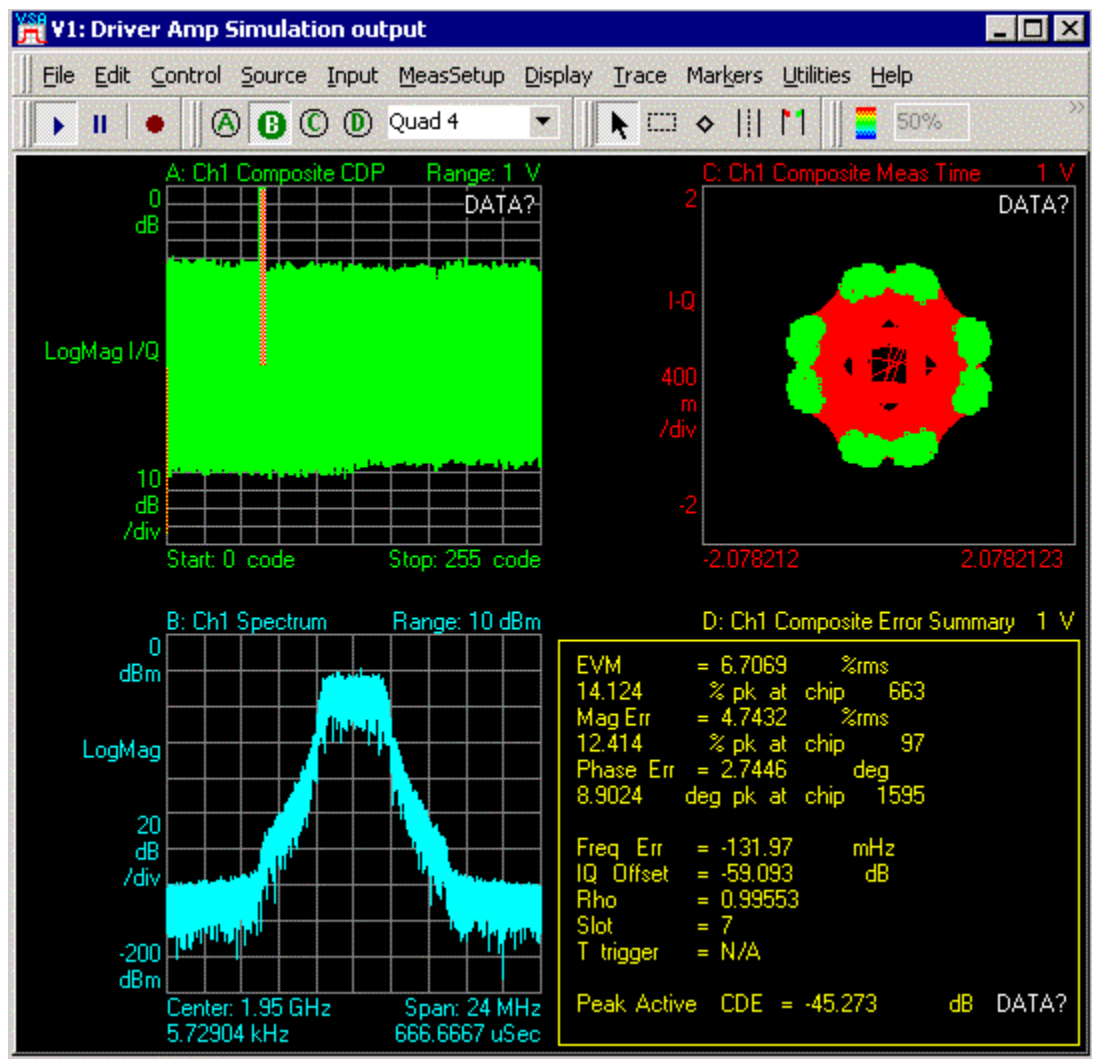

Filling Time Record

INT REF CAL: None

figure 4a : Signaux à l'entrée du PA sous test

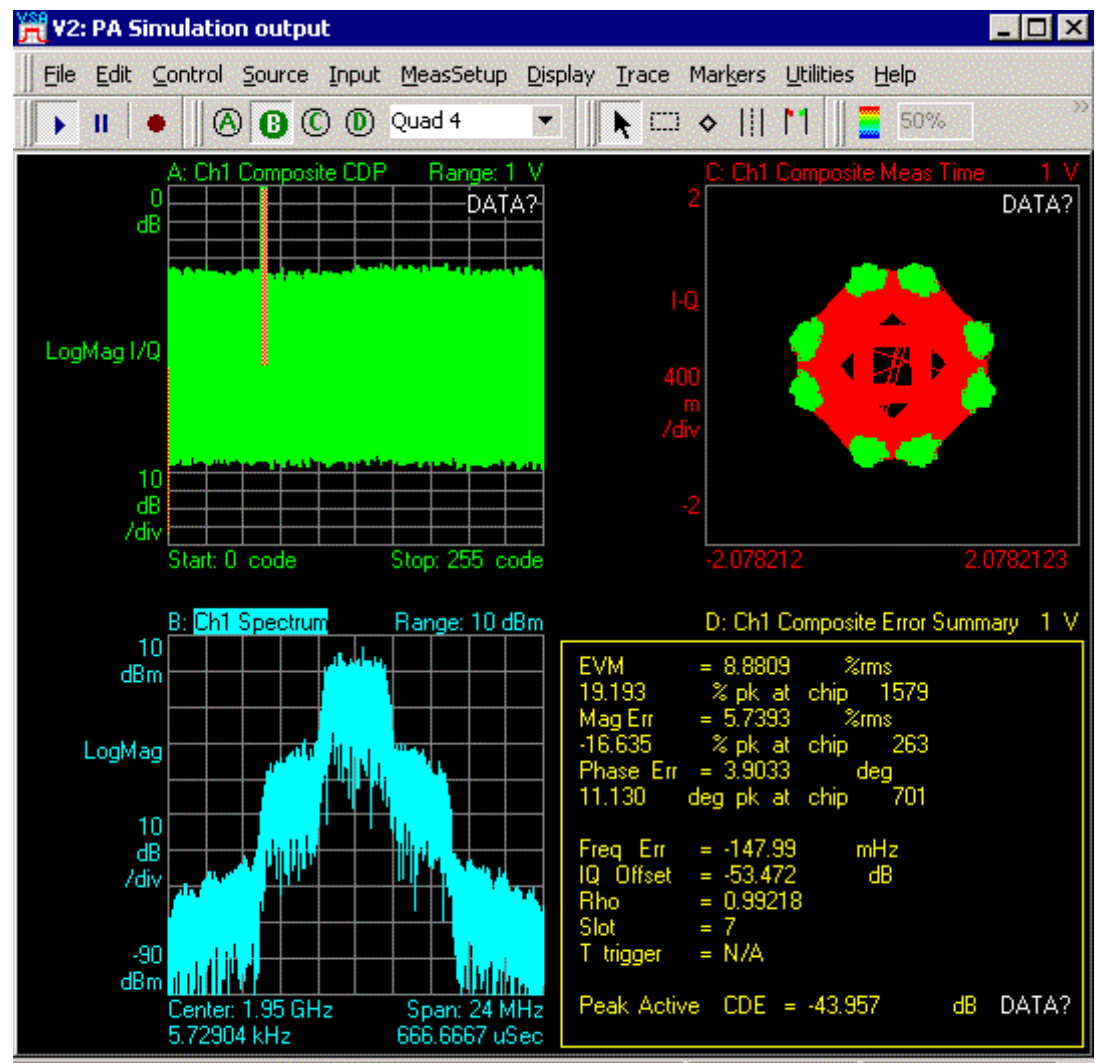

Filling Time Record

INT REF CAL: None

figure $4 \mathrm{~b}$ : Signaux à la sortie du PA sous test 


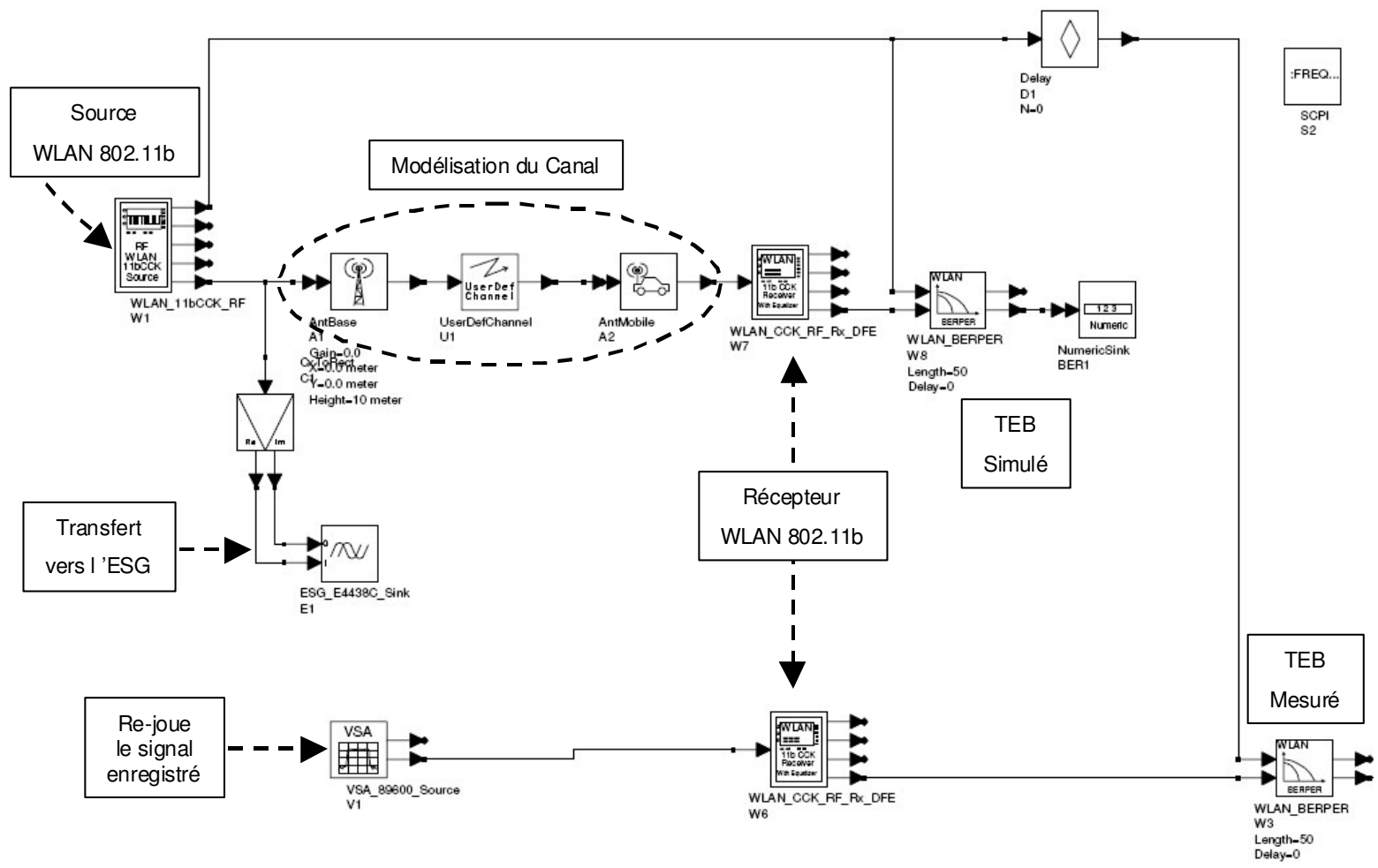

figure 5 : Exemple de «schematic » sous ADS pour l'évaluation des performances en TEB.

Tous ces exemples montrent les atouts et les potentialités de cette plateforme transversale pour les enseignements de type EEA autour des systèmes de radiocommunications. Bien que l'étude et le test de tout système de radiocommunications doivent suivre la construction classique que propose cette plateforme, la souplesse de cet outil liée à l'interaction aisée entre données simulées et mesurées, son couplage avec le logiciel ADS de conception de circuits RF en font un outil de conception véritablement performant. Enfin, couplée à d'autres bancs de caractérisation de dispositifs et de circuits, cette plateforme peut enrichir les enseignements directement liés à la conception de circuits et/ou systèmes entièrement intégrés ou hybrides.

Nous allons maintenant détailler dans les deux parties suivantes deux types d'étude que nous proposons aux élèves de $5^{\text {ème }}$ année des départements GE et TC et aux étudiants de MASTER (M2). Le travail demandé s'inscrit dans la continuité des enseignements liés aux radiocommunications de $3^{\text {ème }}$ et $4^{\text {ème }}$ années qui présentent les méthodes de conception et de simulation des différents éléments d'un émetteur/récepteur de radiocommunications numériques, aussi bien les éléments RF que les éléments numériques. Il est important de préciser qu'il ne s'agit pas ici, à ce niveau d'étude (élèves-ingénieur ou étudiants-master en fin de cursus) d'initier les étudiants soit à la métrologie radio, soit aux systèmes de radiocommunications, ni même à la plateforme présentée. En effet, ils doivent être en mesure de travailler en autonomie, de concevoir leurs propres blocs et de les associer à ceux disponibles sous ADS (même si, préventivement les enseignants-encadrants ont développé des blocs et modèles pour ne pas nuire au bon déroulement des projets). Enfin, la salle utilisée est accessible en dehors des heures marquées à l'emploi du temps.

\section{Etude d'algorithmes SIMO appliqués aux récepteurs multi-standards}

Un grand intérêt de cette plateforme est donc de pouvoir comparer les résultats obtenus avec un canal radio simulé ou mesuré (comme dans l'exemple de la figure 5). De nombreuses techniques permettent de lutter contre les effets néfastes de ce canal de propagation (atténuation, masquage, évanouissements...). Actuellement, l'ensemble des nouvelles normes de communications propose 
d'améliorer les performances de ces liaisons par le recours à des systèmes à antennes multiples. On désigne par MIMO (Multiple Input Multiple Output) un système basé sur plusieurs antennes à l'émission et à la réception. Cette approche suppose une modification de standard à l'émission, alors qu'une approche SIMO (Single Input Multiple Output) peut s'adapter à toute norme existante en améliorant les performances. Nous allons donc présenter ici une étude de ce type réalisable avec le matériel disponible.

Le principe de traitement SIMO est fondé sur le fait qu'en disposant judicieusement plusieurs antennes sur un même terminal, chacune recevra une copie différente (car n'ayant pas subi les mêmes influences du canal) du signal émis. Dès lors, selon les caractéristiques de la forme d'onde et du type de canal traversé, différents algorithmes des plus simples aux plus sophistiqués peuvent être appliqués pour recombiner ces différentes copies en améliorant au final le signal reçu.

De plus, avec l'apparition du concept de la radio logicielle définie (en anglais Software Defined Radio - SDR) [5], des structures de terminaux capables de fonctionner suivant plusieurs modes de transmissions implantés sous forme logicielle en fonction de l'environnement sont très prometteuses. En particulier, il semble intéressant d'ajouter au concept de SDR [6] les capacités de traitement d'antenne SIMO, en adaptant l'algorithme au standard utilisé. Pour que cette étude soit complète, il est important de confronter les résultats obtenus en simulation et ceux obtenus par la mesure. Le travail des étudiants est ici d'étudier différents algorithmes SIMO et de comparer leurs performances via des simulations sous ADS et des campagnes de mesures sur la plateforme d'étude des systèmes de radiocommunications.

\subsection{Traitements SIMO utilisés}

Les structures SIMO développées sont appliquées aux standards $802.11 \mathrm{~b}$ et $802.11 \mathrm{~g}$ qui permettent d'obtenir des débits importants. En fonction de l'environnement et du standard, tous les traitements SIMO n'entraînent pas la même hausse de performances. En effet, ces deux normes WiFi cohabitent dans la même bande de fréquence mais utilisent des formes d'ondes très différentes qui ne réagissent donc pas de la même façon à la traversée de l'environnement. Dans le cas proposé, quels que soient le standard et le type de diversité considérés, les pondérations sont calculées pour minimiser le critère d'erreur quadratique moyenne (en anglais Minimun Mean-Square Error ou MMSE) entre le signal recombiné et le signal de référence constitué du champ "PLCP preamble » des trames $802.11 \mathrm{~b}$ ou du premier symbole du champ «long preamble » des trames $802.11 \mathrm{~g}$ [7]. Des chaînes de simulation de récepteur $802.11 \mathrm{~b}$ et $802.11 \mathrm{~b}$ à 4 voies ont été développées sous ADS. Le tableau 1 résume les algorithmes utilisés en fonction de l'environnement et des standards de communication.

\begin{tabular}{|l|c|c|}
\hline & $802.11 \mathrm{~b}$ & $802.11 \mathrm{~g}$ \\
\hline Diversité spatiale & SMI - MMSE & SMI - MMSE \\
\hline Diversité temporelle & RAKE - 2D & X \\
\hline Diversité fréquentielle & $\mathrm{X}$ & SF - MMSE \\
\hline
\end{tabular}

Tableau 1 - Traitements SIMO utilisés

Les étudiants doivent bien comprendre que la construction par étalement de spectre des trames $802.11 \mathrm{~b}$ rend judicieuses les techniques basées sur l'utilisation de la diversité temporelle. D'autre part, la construction des trames $802.11 \mathrm{~g}$ basée sur le principe de modulation OFDM les encourage à profiter de la diversité fréquentielle. Dans le cas d'un traitement SMI (Sample Matrix Inversion, inversion matricielle basée sur une séquence de référence dans le signal), seules des pondérations spatiales sont appliquées. Le traitement Rake 2D (récepteur râteau à 2 dimensions) correspond à une égalisation temporelle sur chaque voie avant recombinaison optimale des 4 signaux reçus. Enfin, le traitement SF-MMSE (minimisation spatio-fréquentielle) permet d'appliquer des pondérations différentes sur chaque voie mais identiques pour les même groupes de sous-porteuses 
OFDM. Différentes structures de simulations comprenant ces traitements sont fournies, toutes basées sur le même principe que la chaîne mono-voie de la figure 5, mais associant 4 modélisations de canal indépendantes et regroupant les voies au sein d'un bloc de traitement Matlab. Les étudiants peuvent ainsi cerner les nombreux paramètres à optimiser en fonction du standard et du canal de propagation. On présente ici quelques résultats obtenus en termes de TEB (mais bien entendu les autres critères présentés précédemment peuvent être observés). Plus précisément, les courbes suivantes donnent ce TEB en fonction du rapport entre l'énergie moyenne par bit d'information et la densité spectrale d'un bruit blanc $(\mathrm{Eb} / \mathrm{No})$, conduisant les élèves à comprendre l'influence en termes de choix des modulations utilisées.

Les étudiants peuvent ainsi appréhender à la fois les impacts du canal de propagation, mais également le profit que l'on peut tirer de l'utilisation de plusieurs antennes pour lutter contre l'effet le plus néfaste : l'évanouissement. On peut également voir la nécessité de bien dimensionner les structures matérielles ou logicielles des récepteurs en fonction du type de canal (profondeur d'égalisation, taille des séquences de référence, taille des trames en fonction de la cohérence...).

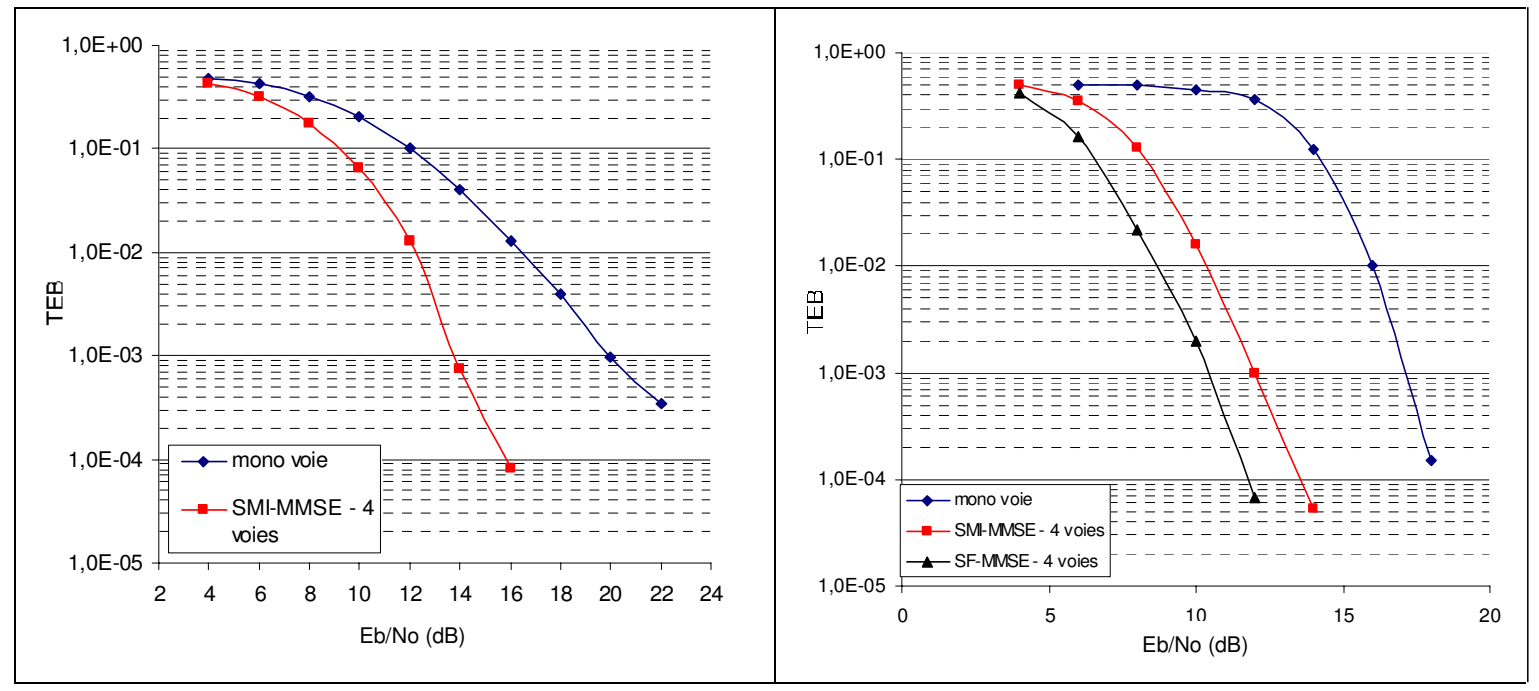

figure 6 : Performances des traitements SIMO - transmission $802.11 \mathrm{~b}$ (gauche) et $802.11 \mathrm{~g}$ (droite)

La figure 6 présente les performances simulées obtenues dans le cas d'une transmission $802.11 \mathrm{~b}$ à $11 \mathrm{Mbps}$ et $802.11 \mathrm{~g}$ à $36 \mathrm{Mbps}$ à travers le canal de propagation ETSI A $\left(\tau_{\mathrm{rms}}=50 \mathrm{~ns}\right.$, étalement temporel de $300 \mathrm{~ns}$ ). Le traitement de diversité spatiale SMI-MMSE est appliqué à chacun des standards, alors que le traitement SF-MMSE (en groupant les fréquences par 4) n'est utilisé que dans le cas de la transmission 802.11g.

\subsection{Comparaison simulation-mesure}

Les systèmes ADS développés permettent de simuler différents types de canaux de propagation relatifs à une transmission à $2,4 \mathrm{GHz}$ proposés par le modèle ETSI [8]. Ces canaux sont tous très dispersifs. Ils présentent de nombreux échos et un étalement temporel relativement important. Ils introduisent donc un facteur de diversité très important. De plus, au cours des simulations, comme il n'est pas possible d'introduire les phénomènes de corrélation de voie, ni de couplage entre antennes, il est constaté par les élèves une surévaluation de l'augmentation des performances obtenues grâce aux traitements SIMO. Une évaluation dans un contexte de fonctionnement le plus réel possible (couplage d'antenne, corrélation de canal, canal de propagation plus ou moins dispersif) apparaît clairement nécessaire.

Des mesures préliminaires sont réalisées grâce à la plateforme radio au sein de nos locaux pour caractériser le canal de propagation qui est celui à notre disposition (banc équivalent à celui de la figure 1, mais avec deux voies de réception sur l'analyseur et deux jeux de mesures pour obtenir 
4 acquisitions exploitables). Ce travail permet aux élèves de conclure quant à la fiabilité de certains modèles de canaux. Il est à noter ici, pour la majorité des pièces où les mesures ont été effectuées, que nous disposons d'un canal à étalement temporel beaucoup plus faible que ceux proposés par l'ETSI. Deux environnements de test ont été utilisés : une configuration de transmission proche de l'AWGN (Additive White Gaussian Noise), et une configuration de transmission à faible étalement temporel ( $\tau_{\mathrm{rms}}=48 \mathrm{~ns}$, étalement temporel de $80 \mathrm{~ns}$, bande de cohérence de 5,8 MHz).

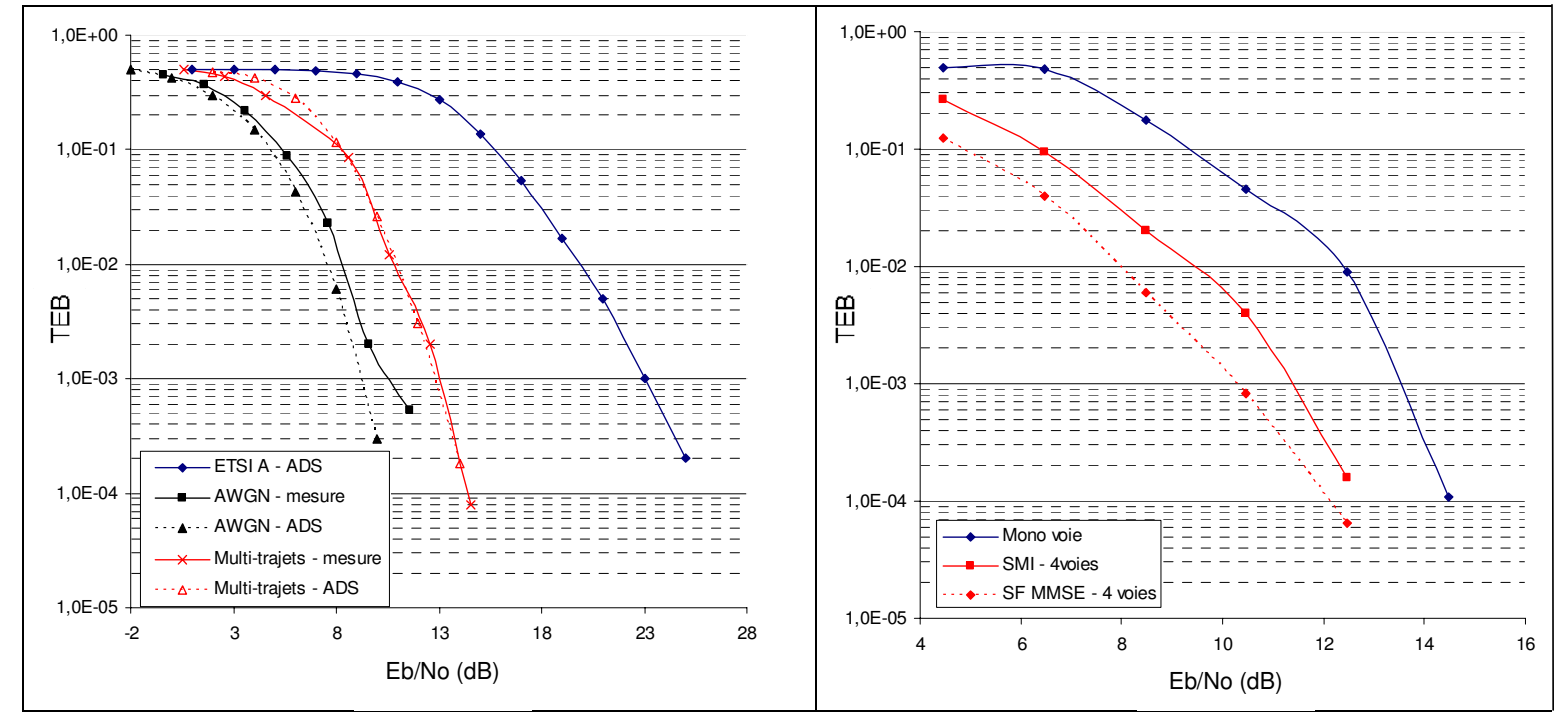

figure 7 : TEB vs. Eb/No - 802.11b mono antenne (gauche) et $802.11 \mathrm{~g} 4$ antennes (droite)

Sur la figure 7, nous avons reporté différents résultats de TEB en fonction de Eb/No. Les données simulées ont été obtenues en étudiant une transmission $802.11 \mathrm{~b}$ à $11 \mathrm{Mbps}$ et une transmission $802.11 \mathrm{~g}$ à $36 \mathrm{Mbps}$. Les calculs de TEB se font sur 10000 trames de 100 octets chacune. Les 4 voies sont enregistrées l'une après l'autre et aucun phénomènes de couplage ou de corrélation spatiale n'est introduit. Une première étape de validation proposée correspond à l'étude de performances des structures de réception mono-antenne développées sous ADS pour différents canaux de propagation. Les étudiants sont amenés à vérifier le très faible écart entre les performances simulées et mesurées en environnement AWGN $(<1 \mathrm{~dB})$ et la correspondance des résultats en environnement multi-trajets pour une transmission $802.11 \mathrm{~b}$. En outre, cette figure permet de mettre en évidence que le canal multi-trajets utilisé pour les mesures est bien moins sélectif que le canal ETSI A représentatif d'un milieu indoor dense, et offre donc peu de diversité (car moins de trajets multiples).

La seconde figure présente l'amélioration des performances obtenues grâce aux traitements SMI et SF-MMSE appliquées au récepteur $802.11 \mathrm{~g}$. Un gain de diversité spatiale de $3 \mathrm{~dB}$ et un gain de diversité spatio-fréquentielle d'environ $4 \mathrm{~dB}$ est clairement observé. Ces valeurs sont en dessous des valeurs théoriquement atteignables (cf. figure 6), mais elles sont dues à la très faible sélectivité du canal.

\section{Etude des limitations RF sur les performances du récepteur 802.11g}

Cette étude, qui s'étale sur un semestre entier, s'inscrit dans la partie dédiée aux éléments RF d'une liaison radio où la conception des circuits analogiques, RF et microondes et le choix de l'architecture (et son dimensionnement) du front-end associé (architectures superhétérodyne, homodyne et faible FI -fréquence intermédiaire-) sont au centre de cet enseignement. Pour les élèves-ingénieurs des départements GE et TC de l'INSA, le travail demandé fait suite à l'étude d'algorithmes SIMO précédemment présentée. 
Dans cette $4{ }^{\text {ème }}$ partie, nous abordons donc l'impact des imperfections radiofréquences sur les performances globales d'un récepteur SIMO-OFDM pour le standard 802.11g. En particulier, le travail demandé aux étudiants est d'étudier l'influence du déséquilibre des voies I et $\mathrm{Q}$ en gain et en phase, le bruit de phase de l'oscillateur local ainsi que les non-linéarités de l'amplificateur de puissance utilisé au niveau de l'émetteur. Les performances du système conçu et simulé sous le logiciel ADS sont évaluées en termes de taux d'erreur par bit (TEB), de diagramme de constellation des états pour chaque sous porteuse, et d'évolution de la densité spectrale de puissance du signal. Les résultats présentés concernent des systèmes mono-antenne et quatre antennes (au niveau du récepteur). L'algorithme de traitement est le SMI et deux types de modèle de canaux de propagation, AWGN et multi-trajets, font également partis des conditions de base de cette étude. Dans l'étude proposée, les étudiants ont à leur disposition la chaîne d'émission-réception sous Ptolemy qui est déjà fonctionnelle mais exempt de défauts RF. Ils ont par ailleurs de nombreux articles et ouvrages à leur disposition (dont certains sont reportés ci-après).

\subsection{Bruit de phase de l'oscillateur local}

Il a été montré dans les systèmes mono-antenne que l'influence du bruit de phase d'un oscillateur local sur les performances du récepteur peut être séparée en deux parties [9]. Une contribution dite «erreur de phase du signal » considérée comme du bruit additif sur la phase du signal. Cette erreur est causée par le bruit de phase près de la porteuse. La seconde contribution est liée à une dégradation du bruit dans la bande du signal par mélange du bruit de phase loin de la porteuse avec un signal interférent fort. Pour quantifier ces effets sur le TEB, dans le cas du récepteur $802.11 \mathrm{~g}$, nous faisons développer aux étudiants des modèles de bruit de phase soit via le paramètre PhaseNoiseData de la source N-Tones du simulateur d'ADS-Ptolemy soit des modèles plus élaborés en langage $C$ (pour des modèles plus physiques intégrant par exemple le modèle de Leeson [1]) permettant de quantifier l'effet des composantes de bruit BF de l'amplificateur intégré dans l'oscillateur (bruit thermique, en $1 / \mathrm{f} . .$. ), leur niveau en $\mathrm{dBc} / \mathrm{Hz}$ ou encore la présence de raies parasites (spurious) en sortie de la PLL (Phase locked loop).

Nous avons reporté sur la figure 8 , les performances en terme de TEB en fonction de Eb/No dans les cas de récepteur SISO et SIMO-OFDM (1 et 4 antennes) avec un canal AWGN pour modéliser la propagation radio Emetteur-Récepteur. Pour les courbes présentées, c'est le bruit en 1/f qui sert de référence au modèle de bruit de phase de l'oscillateur local comme indiqué ci-après. Nous avons utilisé ici le paramètre «PhaseNoiseData». PN1, PN2 et PN3 sont les spécifications de l'oscillateur local (OL) en bruit de phase retenues pour ces simulations.

$$
\begin{aligned}
& \text { PN1 (-口-) : } \quad \text { pas de bruit de phase } \\
& \text { PN2 (-O-) : } \quad-60 \mathrm{dBc} / \mathrm{Hz} \text { à } 100 \mathrm{~Hz} \\
& -90 \mathrm{dBc} / \mathrm{Hz} \text { à } 1 \mathrm{kHz} \\
& -150 \mathrm{dBc} / \mathrm{Hz} \text { à } 100 \mathrm{kHz} \\
& \text { PN3 }\left(-\Delta_{-}\right): \quad-20 \mathrm{dBc} / \mathrm{Hz} \text { à } 100 \mathrm{~Hz} \\
& -50 \mathrm{dBc} / \mathrm{Hz} \text { à } 1 \mathrm{kHz} \\
& -110 \mathrm{dBc} / \mathrm{Hz} \text { à } 100 \mathrm{kHz}
\end{aligned}
$$

Sur la figure 8, il est clair que le bruit de phase dégrade sensiblement les performances en taux d'erreur par bit. Pour des niveaux de Eb/No supérieur à $8 \mathrm{~dB}$ on constate une variation d'au moins une décade (de l'ordre de $3.10^{-2}$ avec PN1 et de $3.10^{-1}$ avec PN3) pour le cas $1 \times 4$ AWGN. Pour pouvoir aller plus loin dans l'analyse, c'est à dire dans la comparaison des récepteurs SISO et SIMO et dans la compensation éventuelle des effets du bruit de phase observés, il est utile de tracer ces résultats en relatif. Sur la figure 9 nous avons reporté cette comparaison en TEB relatif des deux 
types de récepteur en fonction du niveau de bruit de phase de l'OL et pour les deux modèles de canal : le modèle AWGN et modèle A multi-trajets d'ADS. La référence prise est la valeur de TEB

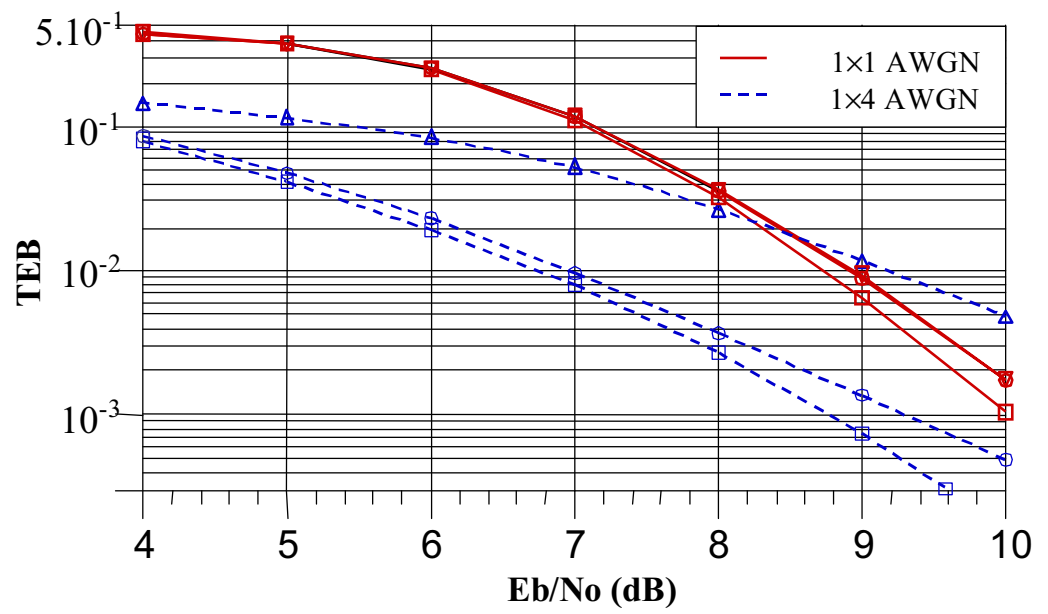

figure 8 : TEB en fonction de $\mathrm{Eb} / \mathrm{No}$ (canal AWGN)

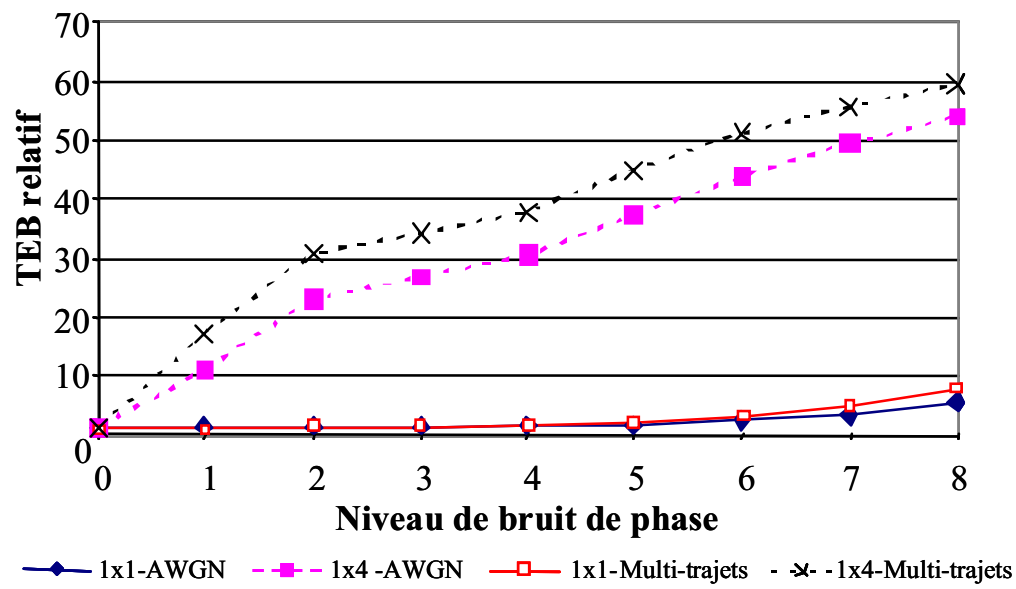

figure 9 : TEB relatif en fonction du niveau de bruit de phase (“0” aucun;“" 8 "- $40 \mathrm{dBc} / \mathrm{Hz}$ à $100 \mathrm{~Hz}$ )

du système 1 antenne sans bruit de phase appliqué. On peut constater que, quel que soit le canal utilisé, cette dégradation des performances est importante et largement augmenté pour le système 4 antennes. En conséquence, l'algorithme SMI n'apparaît pas capable de compenser les effets de l'augmentation du bruit de phase de l'oscillateur local.

Pour conclure sur cette partie, il est important de signaler que le simulateur ADS qui permet de bien quantifier l'impact d'un bruit de phase proche de la porteuse ne permet pas actuellement de travailler à des fréquences d'offset supérieures au $\mathrm{MHz}$ (les résultats observés en simulation sont très variables suivant les paramètres de simulation et donc peu exploitables). Ceci est évidemment préjudiciable à l'étude et à la conception de systèmes OFDM où les problèmes hors bandes peuvent être très présents.

\subsection{Non-linéarités de l'amplificateur de puissance de l'émetteur}

Il est également important d'étudier l'impact des distorsions harmoniques et d'intermodulation sur les performances du récepteur. Les spécifications sont d'ailleurs sévères dans les systèmes de communications au niveau des émissions parasites. Dans le cas de modulations à enveloppe non- 
constante l'importance de la linéarité du PA [10] est capitale pour éviter l'augmentation du niveau des lobes secondaires du spectre du signal en sortie de l'amplificateur (cf. figures $4 \mathrm{a}$ et $4 \mathrm{~b}$ du paragraphe 2). Pour le système $802.11 \mathrm{~g}$ étudié, les étudiants peuvent utiliser des modèles simples prenant en compte le point de compression à $1 \mathrm{~dB}\left(\mathrm{P}_{1 \mathrm{~dB}}\right)$ et le point d'interception d'ordre 3 (IP3 ou $T O I$ en anglais) via le bloc Gain RF d'ADS-Ptolemy (figure 10). En effet, à partir de cet élément et plus particulièrement avec le paramètre GCType (qui permet de modéliser la compression du gain de l'amplificateur), on peut rapidement tenir compte (figure 11) du point $\mathrm{P}_{1 \mathrm{~dB}}$, de 1 'IP3, de la puissance de saturation ou encore travailler à partir des courbes $\mathrm{AM} / \mathrm{AM}$ et $\mathrm{AM} / \mathrm{PM}$. Il est à noter que chacun de ces paramètres est référencé sous Ptolemy par rapport à la sortie du bloc Gain RF.

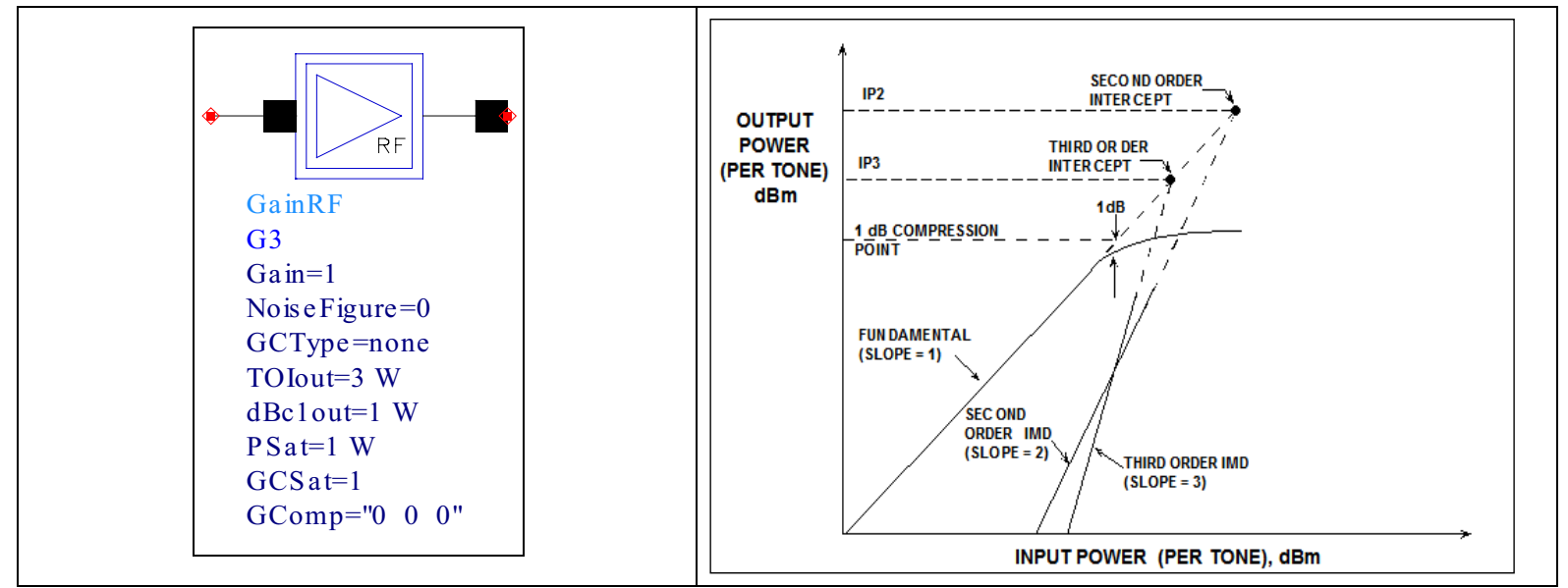

figure 10: Bloc d'ADS-Ptolemy pour les modèles d'amplificateurs figure 11 : Compression du gain d'un amplificateur (figure issue de l'aide en ligne d'ADS)

Les études effectuées utilisent les conditions de simulation identiques au paragraphe 4.1. Sur la figure 12 , les effets du point $\mathrm{P}_{1 \mathrm{~dB}}$ et du TOI sont reportés $(\mathrm{Eb} / \mathrm{No}=10 \mathrm{~dB})$ dans le cas du canal multitrajets. C'est le modèle GCTypel prenant en compte seulement le point $\mathrm{P}_{1 \mathrm{~dB}}$ qui a été utilisé pour étudier l'influence du point de compression à $1 \mathrm{~dB}$. Par contre, pour l'étude du point d'interception d'ordre 3, c'est le modèle GCType2 prenant en compte simultanément le point $\mathrm{P}_{1 \mathrm{~dB}}$ et le TOI avec une différence de l'ordre de $10 \mathrm{~dB}$ que l'on doit intégrer sous ADS-Ptolemy. Les résultats présentés sur la figure 12 montrent aux étudiants que pour un $\mathrm{P}_{1 \mathrm{~dB}}$ ou un TOI fixé, le système SIMO est sensiblement plus performant que le système SISO. Par ailleurs, ces études leur ont permis également de mettre en évidence le bénéfice apporté par le SMI pour le système 4 antennes dans le cas du canal multi-trajets par rapport au canal AWGN (résultats non reportés).

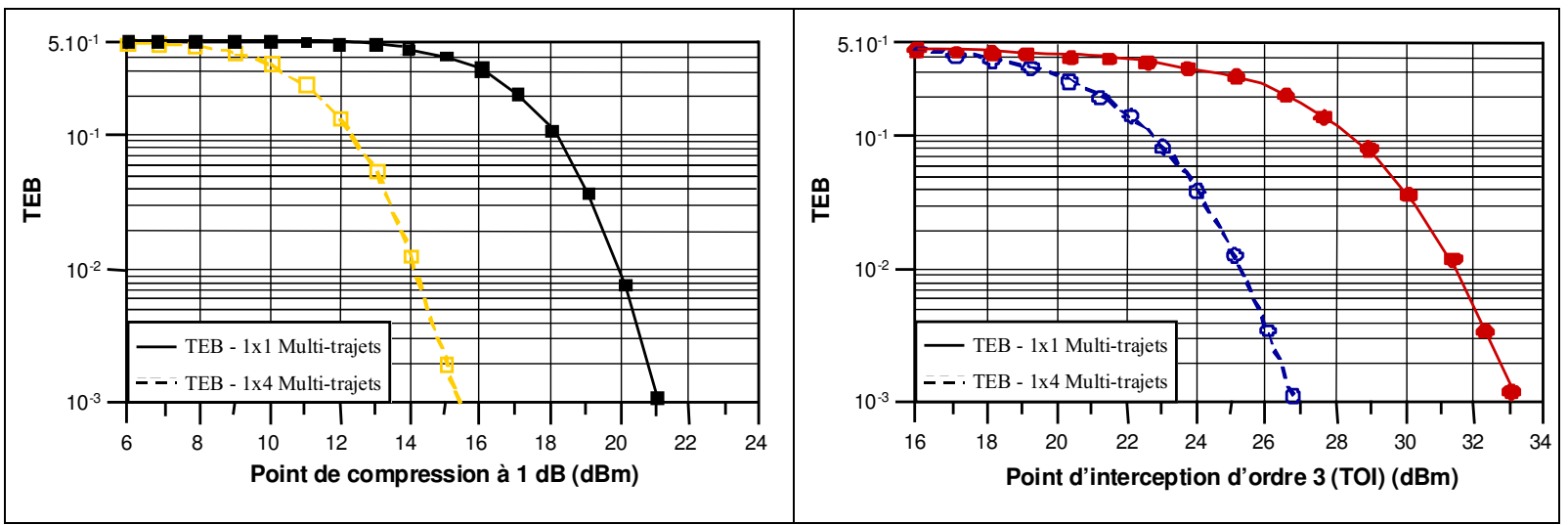

figure 12 : TEB en fonction du point $\mathrm{P}_{1 \mathrm{~dB}}$ (gauche) du TOI (droite)

(Gain linéaire du $\mathrm{PA}=10 \mathrm{~dB}$ et $\mathrm{Eb} / \mathrm{No}=10 \mathrm{~dB}$ ) 
Sur la figure 13, les deux cas 1 et 4 antennes sont comparés qualitativement à partir du diagramme des constellations. Les points de la constellation apparaissent plus « bruités » dans la cas du récepteur mono-antenne.

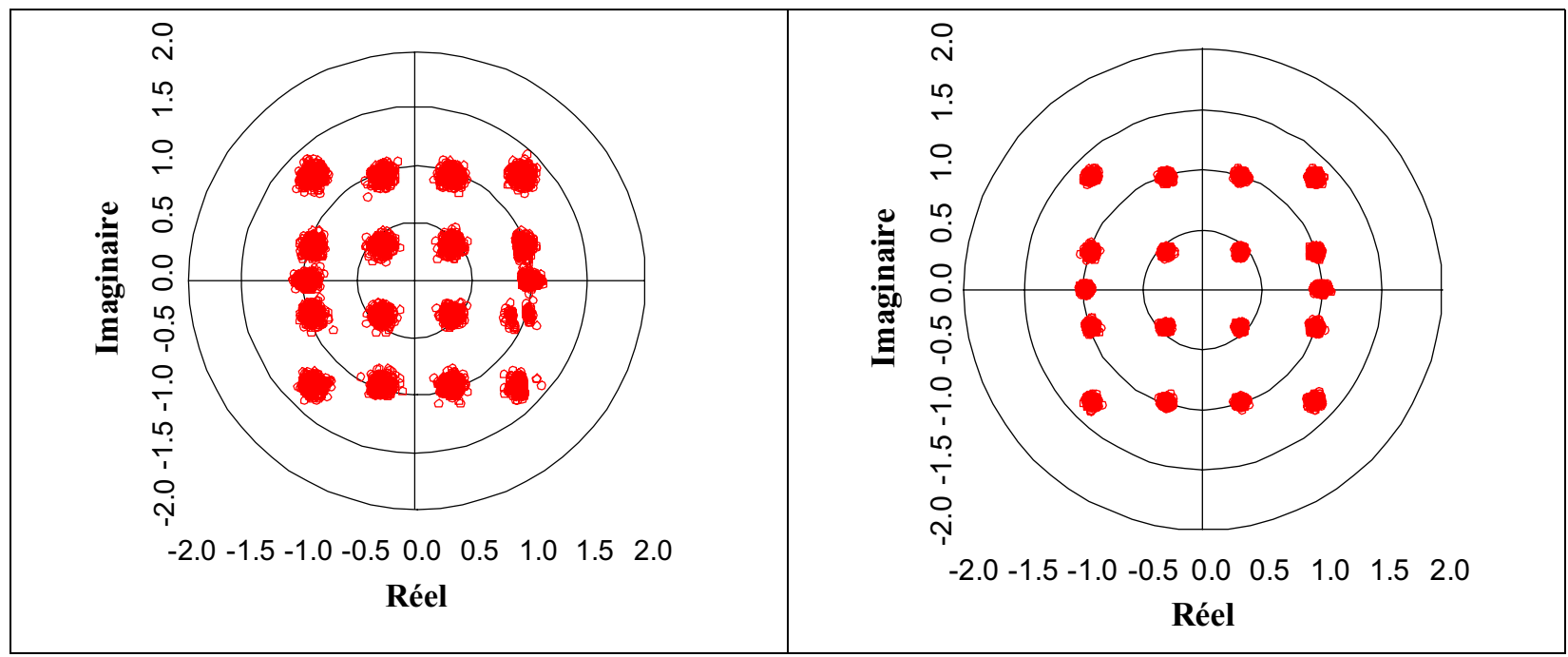

figure 13 : Constellation QAM 16 états pour le récepteur 1 antenne (gauche) et 4 antennes (droite) Cas du canal AWGN avec TOI $=46 \mathrm{dBm}$, Gain linéaire $=20 \mathrm{~dB}$

\subsection{Déséquilibre des voies I et $\mathrm{Q}$}

Le déséquilibre des voies I et $\mathrm{Q}$ [11][12] est un point crucial dans un récepteur en conversion directe. La non idéalité (appariement des 2 voies) de ce bloc qui se situe juste après l'amplificateur faible bruit au niveau de la réception se traduit par une "différence " de phase et d'amplitude additionnelles pour les signaux des deux voies. Ces défauts des voies I et $\mathrm{Q}$ ont pour effet la rotation de la constellation, l'EVM est donc dégradé ainsi que le TEB. Sous ADS les deux paramètres "gain imbalance» et "phase imbalance» du bloc démodulateur QAM (traduisant respectivement le déséquilibre de gain et phase des voies IQ) permettent la prise en compte de ce déséquilibre. La mise en équation est décrite ci-après dans le cas du bloc utilisant un oscillateur local externe pour démoduler le signal $R F . I_{1}(t)+Q_{1}(t)$ représente le signal entrant $R F$ à démoduler et $I_{2}(t)+Q_{2}(t)$ l'enveloppe complexe (représentation en bande de base) du signal de l'oscillateur local introduit dans le bloc démodulateur QAM. Les deux sorties du bloc sont $\mathrm{V}_{\mathrm{I}}(\mathrm{t})$ (en phase) et $\mathrm{V}_{\mathrm{Q}}(\mathrm{t})$ (en quadrature) sont données par les expressions suivantes :

$$
\mathrm{V}_{\mathrm{I}}(\mathrm{t})=\mathrm{S}\left(\mathrm{I}_{1}(\mathrm{t}) \times \mathrm{I}_{2}(\mathrm{t})+\mathrm{Q}_{1}(\mathrm{t}) \times \mathrm{Q}_{2}(\mathrm{t})\right) / \mathrm{A} \quad \text { et } \quad \mathrm{V}_{\mathrm{Q}}(\mathrm{t})=\mathrm{S} \times \mathrm{AIQ} \times\left(\mathrm{I}_{1}(\mathrm{t}) \times \mathrm{VI}+\mathrm{Q}_{1}(\mathrm{t}) \times \mathrm{VQ}\right) / \mathrm{A}
$$

où :

$\mathrm{S}$ est la sensibilité du récepteur, A l'amplitude du signal de l'oscillateur local, $\mathrm{AIQ}=10^{\text {Gain Imbalance/20 }}$ et VI et VQ par les équations ci-dessous :

$\mathrm{VI}=\mathrm{I}_{2}(\mathrm{t}) \times \cos \Phi-\mathrm{Q}_{2}(\mathrm{t}) \times \sin \Phi$

$\mathrm{VQ}=\mathrm{I}_{2}(\mathrm{t}) \times \sin \Phi+\mathrm{Q}_{2}(\mathrm{t}) \times \cos \Phi$

où $\Phi$ est rattaché au paramètre «phase imbalance » par :

$\Phi=\frac{\pi}{2}-$ Phase Im balance $\times \frac{\pi}{180}$

Les résultats en TEB relatif sont présentés sur les figures 14 et 15 pour les deux systèmes avec un canal multi-trajets. La référence 0 de variation en gain et en phase est obtenu pour un SNR de $10 \mathrm{~dB}$ et de $6 \mathrm{~dB}$ respectivement pour les systèmes SISO et SIMO-4 antennes. Pour chacun des 
points des courbes 1000 trames OFDM avec 100 bytes par trame sont les conditions de simulation. On peut constater sur les deux figures que la dégradation des performances en TEB avec l'augmentation des paramètres "gain imbalance » et "phase imbalance » est largement plus importante dans le cas du récepteur 1 antenne.

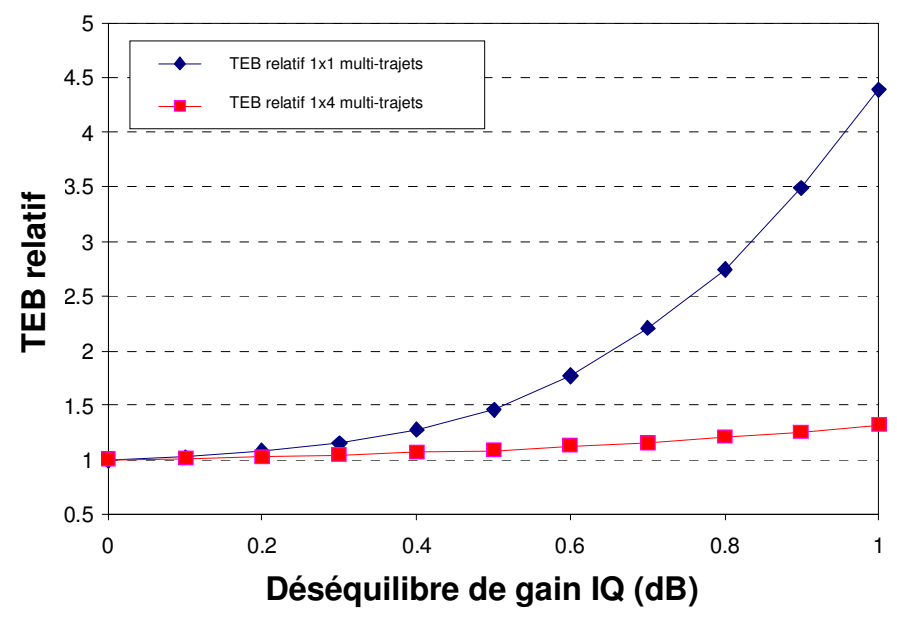

figure 14: TEB relatif en fonction du déséquilibre de gain

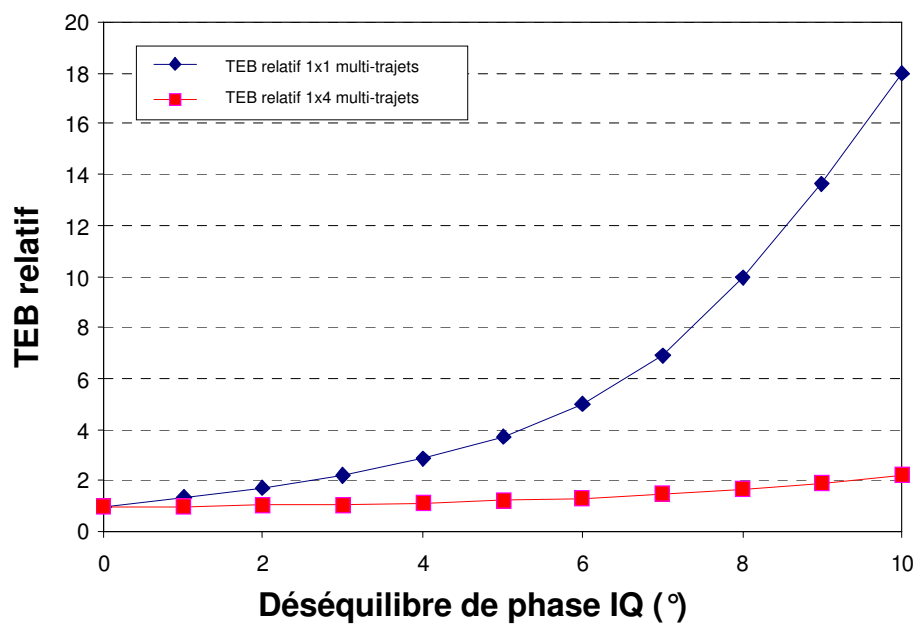

figure 15: TEB relatif en fonction du déséquilibre de phase

Cette étude comparative amène les étudiants à vérifier l'efficacité de techniques de diversité spatiales quant à leur compensation des déséquilibres en gain comme en phase des voies de sortie du démodulateur QAM, y compris pour l'algorithme SMI utilisé ici.

\section{Conclusion}

Une approche globale de conception associant l'utilisation d'antennes multiples, la technique d'OFDM et les principes de SDR est un choix reconnu comme réellement judicieux aujourd'hui pour l'analyse et la conception de futurs systèmes sans fils larges bandes. Cette nécessité, initialement associée aux problématiques $R \& D$ de conception de réseaux de capteurs ou de conception hétérogène de systèmes sur puce se généralise aujourd'hui. En conséquence, le vaste domaine des radiocommunications se doit d'être enseigné à nos futurs ingénieurs à hauteur des défis et enjeux de cette révolution technologique. L'utilisation conjointe d'outils logiciels et matériels performants 
devient alors nécessaire pour les formations universitaires. Notre plateforme d'étude, de conception et de test des systèmes de radiocommunications qui donne la possibilité de faire le lien entre les technologies microélectroniques, les composants actifs et passifs et les circuits clés RF répond largement à ces nouveaux besoins de formation.

Les deux sujets de travail proposés aux étudiants en fin de cursus sur les algorithmes SIMO ou sur l'impact des limitations RF sur les performances d'un récepteur sont des points clés que les étudiants doivent maîtriser dans une démarche de conception de systèmes de radiocommunications multiantennes et multi-normes. A ces projets, pour quelques étudiants, succèdent des projets de fin d'étude sur la même thématique. En particulier, ils sont amener à approfondir l'influence des défauts RF sur différentes architectures de récepteur (superhétérodyne, faible FI), en ayant une approche plus précise c'est à dire quant aux plages de variation des paramètres RF critiques, plus en lien avec une technologie semiconducteur donnée ou encore en cumulant les effets des différents blocs étudiés. Cette démarche permet à ces étudiants d'évaluer les contraintes RF les plus critiques et celles que l'on peut relâcher en les quantifiant précisément. Il est clair que ce type d'étude n'est pas réalisable dans l'emploi du temps d'une promotion complète d'étudiants et que c'est seulement dans le cadre de projets de fin d'études ou de stages de recherche que l'on peut approfondir ces travaux.

\section{Références bibliographiques}

[1] G. Baudoin, M. Villegas et al. " Radiocommunications numériques, tome 1 et 2 » Ed. Dunod, 623 p. et 446 p., 2002.

[2] R. Alison "Software Radio: A Modern Approach to Radio Engineering " Ed. Prentice Hall, 592 p., 2002.

[3] http://eesof.tm.agilent.com/products/ads main.html

[4] P.F. Morlat, H. Parvery, G. Villemaud, J. Verdier, J.M. Gorce, " Global System Evaluation Scheme for Multiple Antennas Adaptive Receivers ", European Conference on Wireless Technology, Manchester, sept. 2006.

[5] J. Mitola, " The Software radio architecture », IEEE Communication Magazine, mai 1995

[6] X. Xu, R. Bosisio, K Wu, "Analysis and Implementation of software defined radio receiver platform », APMC, Suzhou, 2005

[7] P.F. Morlat, P. Mary, G. Villemaud, J.M. Gorce, M. Arndt, "Performance Validation of a Multi-Standard and Multi-Antenna Receiver », European Conference on Antennas and Propagation, Nice, nov. 2006.

[8] Channel Models for HIPERLAN/2 in different indoor Scenarios. ETSI EP BRAN 3ER1085B, 1998.

[9] S. Wu et al. "Performances analysis on the effect of phase noise in OFDM systems », Proc. IEEE 7th international symposium on Spread Spectrum Techniques and Applications, vol. 1, pp. 133-138, sept. 2002.

[10]F.H. Gregorio and T.J. Laakso, "The performance of OFDM-SDMA systems with power amplifier non-linearities », Proc.2005 Finnish Signal Processing Symposium, pp. 58 - 61, août 2005.

[11]M. Brandolini et al. "Toward Multistandard Mobile Terminals - Fully Integrated Receivers Requirements and Architectures ", IEEE Transaction on Microwave Theory and Techniques, vol. 53, No 3, mars 2005

[12] J. Tubbax et al. "Joint Compensation of IQ imbalance, Frequency Offset and Phase Noise in OFDM Receivers ", European Transaction on Telecommunications, Special Issue on Multi-Carrier Spread-Spectrum, vol. 15, mai 2004. 